\title{
A Continued Fraction Expansion for a Generalization of Dawson's Integral
}

\author{
By D. Dijkstra
}

\begin{abstract}
A continued fraction expansion for a generalization of Dawson's integral is presented. An exact formula for the truncation error in terms of the confluent hypergeometric function is derived. The expansion is shown to have good convergence properties for both small and large values of the argument.
\end{abstract}

I. Introduction. The function

$$
F(p, x)=e^{-x} \int_{0}^{x} e^{t^{p}} d t, \quad(x \geqslant 0, p>0)
$$

is a generalization of Dawson's integral

$$
F(2, x)=e^{-x^{2}} \int_{0}^{x} e^{t^{2}} d t
$$

A graphical representation of $F(p, x)$ for $p=2(1) 6$ and a table of values of $F(2, x)$ can be found in Abramowitz and Stegun [1]. The function $F(3, x)$ occurs in viscous fluid mechanics and $F\left(1 / n, z^{n}\right)$ is (apart from a numerical factor) an exact representation of the remainder in the $n$-term Taylor series for $\exp (-z)$. Dawson's integral arises frequently in several branches of physics and various numerical methods have been developed for its computation. Wynn [3] obtained a particular continued fraction expansion for $F(2, x)$ which appeared to converge fast for both small and large positive values of $x$. This property was further exploited by McCabe [2] who gave an exact expression for the truncation error of this continued fraction for Dawson's integral. He also discussed other methods used in the past for the computation of the function (1.2).

In this paper we will generalize the results of Wynn [3] and McCabe [2] to arbitrary positive values of $p$. In other words we will derive the truncation error of a particular continued fraction expansion for the function (1.1). The expansion will be shown to converge fast for both small and large positive values of the argument. Further, a table is provided showing the number of convergents needed for the evaluation of $F(p, x)$ with an accuracy of 10 significant digits.

II. Expansions of $F(p, x)$. The following notation will be used:

$$
z=x^{p}, \quad a=1 / p, \quad(x \geqslant 0, p>0) .
$$

The function $M(\alpha, \beta, z)$ denotes the confluent hypergeometric function in the notation of Abramowitz and Stegun [1], viz.

Received April 27, 1976; revised September 14, 1976.

AMS (MOS) subject classifications (1970). Primary 41 A20, 30A22. tion error.

Key words and phrases. Continued fractions, confluent hypergeometric functions, trunca-

Copyright $\odot 1977$, American Mathematical Society 


$$
\begin{gathered}
M(\alpha, \beta, z)=\sum_{n=0}^{\infty} \frac{(\alpha)_{n}}{(\beta)_{n}} \frac{z^{n}}{n !}, \quad \beta \neq 0,-1,-2, \ldots ; \\
(\alpha)_{n}=\alpha(\alpha+1) \cdots(\alpha+n-1), \quad(\alpha)_{0}=1 .
\end{gathered}
$$

Expanding the integrand of (1.1), we obtain in terms of the variables (2.1) with the aid of Kummer's transformation

$$
F(p, x)=x e^{-z} M(a, a+1, z)=x M(1, a+1,-z) .
$$

The convergent Taylor series of $F(p, x)$ is given by

$$
F(p, x)=x \sum_{n=0}^{\infty} \frac{(-z)^{n}}{(a+1)_{n}}
$$

and its divergent asymptotic expansion at infinity reads

$$
F(p, x) \sim a x \sum_{n=0}^{\infty}(1-a)_{n} z^{-n-1}, \quad z=x^{p} \rightarrow \infty .
$$

The Taylor series (2.4) can be used to compute $F$ for small values of $z$; but as $z$ becomes large, a disastrous loss of digits is encountered. The expansion (2.5) has also its limitations since the development diverges for all $z$ if $a \neq 1,2,3, \ldots$; hence it is useful only if $z$ is sufficiently large.

Corresponding to each individual expansion (2.4) and (2.5), there exist continued fraction expansions which, for the case $p=2$ (Dawson's integral), were given by McCabe [2]. As regards numerical properties, these continued fractions have virtually the same limitations as the expansions to which they correspond. A third continued fraction expansion for $F(p, x)$ is

$$
F(p, x)=\frac{a x}{a+z}-\frac{z}{a+1+z}-\frac{2 z}{a+2+z}-\cdots \frac{n z}{a+n+z} \cdots,
$$

$$
z=x^{p}, \quad a=\frac{1}{p},
$$

and this expansion corresponds to both the Taylor series (2.4) and the asymptotic expansion (2.5). The special case $p=2$ was considered by McCabe [2]. The expansion (2.6) is certainly convergent for the values of $z$ considered here (i.e. $z \geqslant 0$ ) and the convergence is fast for small as well as large values of $z$. In Section 4 we will give an elementary derivation with truncation error of the expansion (2.6). The proof is based on properties of the function $M(\alpha, \beta, z)$.

III. Properties of $M(\alpha, \beta, z)$. The confluent hypergeometric function $M$ satisfies the recurrence relations

$$
\begin{aligned}
& \beta(\beta-1) M(\alpha, \beta-1, z)+\beta(1-\beta-z) M(\alpha, \beta, z)+z(\beta-\alpha) M(\alpha, \beta+1, z)=0 \\
& \beta(\beta-1) M(\alpha-1, \beta-1, z)+\beta(1-\beta+z) M(\alpha, \beta, z)-\alpha z M(\alpha+1, \beta+1, z)=0
\end{aligned}
$$

Differentation of $M(\alpha, \beta, z)$ with respect to $z$ can easily be performed as follows

$$
M^{\prime}(\alpha, \beta, z)=\alpha M(\alpha+1, \beta+1, z) / \beta \text {. }
$$


Another relation between functions $M$ is

$$
\begin{aligned}
& \beta(\beta-1) M(\alpha, \beta,-z) M(-\alpha, 1-\beta, z) \\
& -\alpha z M(1-\alpha, 2-\beta, z) M(\alpha+1, \beta+1,-z)=\beta(\beta-1),
\end{aligned}
$$

where $\beta \neq 0, \pm 1, \pm 2, \ldots$.

The formulas (3.1)-(3.3) can be found in Abramowitz and Stegun [1] but the nonlinear equation (3.4) has not been listed there.

Proof of Eq. (3.4). At $z=0$ the equation is correct. Differentiating the lefthand side of (3.4) with respect to $z$, we find, using (3.3),

$$
(d / d z)(\text { L.H.S. })=-\alpha M(\alpha+1, \beta+1,-z) S+\alpha M(1-\alpha, 2-\beta, z) T /(\beta+1),
$$

where

$$
\begin{aligned}
& S=(\beta-1) M(-\alpha, 1-\beta, z)+M(1-\alpha, 2-\beta, z)+z(1-\alpha) M(2-\alpha, 3-\beta, z) /(2-\beta), \\
& T=\beta(\beta+1) M(\alpha, \beta,-z)+z(\alpha+1) M(\alpha+2, \beta+2,-z) .
\end{aligned}
$$

Rewriting the quantities $S$ and $T$ by means of an adjusted form of (3.2), we obtain

$$
S=(\beta+z) M(1-\alpha, 2-\beta, z) ; \quad T=(\beta+1)(\beta+z) M(\alpha+1, \beta+1,-z) .
$$

Substitution of these results in Eq. (3.5) yields

$$
(d / d z)(\text { L.H.S. })=0 \text {, }
$$

which proves (3.4).

IV. The Continued Fraction Expansion. With the notation (2.1) we can write (2.3) as

$$
F(p, x) / x=e^{-z} M(a, a+1, z)=M(a, a+1, z) / M(a, a, z) .
$$

Instead of this particular ratio of two functions $M$, the following generalized expression will be considered:

$$
K(a, b, z)=M(a, b+1, z) / b M(a, b, z), \quad a, b>0, z \geqslant 0 .
$$

The factor $b$ has been inserted for convenience. Throughout this section it is assumed that the variables $a, b$ and $z$ satisfy the inequalities (4.2). In terms of the variables (2.1) the function $K$ satisfies the relations

$$
F(p, x)=a x K(a, a, z)
$$

and

$$
0<K(a, b, z) \leqslant 1 / b
$$

In order to obtain a continued fraction for $K$ we take $\alpha=a$ and $\beta=b+1$ in the recurrence relation $(3.1)$, whence

$$
K(a, b, z)=1 /\{(b+z)-z(b+1-a) K(a, b+1, z)\} .
$$


An immediate consequence of this equation is the finite continued fraction

$$
\begin{aligned}
K(a, b, z)=\frac{1}{b+z}- & \frac{z(b+1-a)}{b+1+z} \\
& \cdots-\frac{z(b+N-1-a)}{b+N-1+z}-\frac{z(b+N-a) K(a, b+N, z)}{1} .
\end{aligned}
$$

The right-hand side is an exact representation of the left-hand side for all $N=1,2$, $3, \ldots$ With $N=\infty$ we get the expansion

$$
K(a, b, z)=\frac{1}{b+z}-\frac{z(b+1-a)}{b+1+z}-\cdots \frac{z(b+n-a)}{b+n+z}-\cdots
$$

This expansion was given by Wynn [3] , but the terminating continued fraction (4.5) contains useful information about the truncation error of Wynn's expansion. In the following the integer $N$ in (4.5) will have a fixed value $N>1$. If $P_{n}$ denotes the numerator and $Q_{n}$ the denominator of the $n$th convergent of the continued fraction (4.5), then

$$
\frac{P_{n}}{Q_{n}}=\frac{1}{b+z}-\frac{z(b+1-a)}{b+1+z}-\cdots \frac{z(b+n-1-a)}{b+n-1+z}, \quad 1 \leqslant n \leqslant N .
$$

The convergent $P_{N} / Q_{N}$ is an approximation to (4.5) and the next convergent is exact:

$$
K(a, b, z)=P_{N+1} / Q_{N+1} .
$$

Hence, the error in the $N$ th convergent is

$$
K(a, b, z)-P_{N} / Q_{N}=P_{N+1} / Q_{N+1}-P_{N} / Q_{N}
$$

Using a well-known result from the theory of continued fractions we find

$$
K(a, b, z)-P_{N} / Q_{N}=(b-a+1)_{N} K(a, b+N, z) z^{N} / Q_{N} Q_{N+1} .
$$

To proceed further we need information concerning the denominators $Q_{N}$ which satisfy the recurrence relation

$$
\left\{\begin{aligned}
Q_{-1} & =0, \quad Q_{0}=1 \\
Q_{n} & =(b+n-1+z) Q_{n-1}-z(b+n-1-a) Q_{n-2} \quad(n=1,2, \ldots, N), \\
Q_{N+1} & =Q_{N}-z(b+N-a) K(a, b+N, z) Q_{N-1} .
\end{aligned}\right.
$$

THEOREM 1. With variables $a, b$ and $z$ in the range (4.2) the functions $Q_{n}$ satisfy

$$
\begin{aligned}
Q_{n} & \geqslant(b)_{n}+z^{n} \quad(n=1,2, \ldots, N), \\
Q_{N+1} & \geqslant(b)_{N}+a z^{N} K(a, b+N, z) .
\end{aligned}
$$

Proof. We show by induction that 


$$
Q_{n} \geqslant z Q_{n-1}+(b)_{n} \quad(n=0,1, \ldots, N) .
$$

For $n=0,1$ this inequality is correct. Assume

$$
Q_{i} \geqslant z Q_{i-1}+(b)_{i} \quad(i=0,1, \ldots, n-1 ; n<N)
$$

then $Q_{i}>0$. Hence

$$
\begin{aligned}
Q_{n} & =z Q_{n-1}+(b+n-1)\left(Q_{n-1}-z Q_{n-2}\right)+a z Q_{n-2} \\
& \geqslant z Q_{n-1}+(b+n-1)(b)_{n-1}=z Q_{n-1}+(b)_{n},
\end{aligned}
$$

which proves (4.14). The inequality (4.12) is an immediate consequence of (4.14). To prove (4.13) we use (4.4), (4.12) and (4.14) to estimate $Q_{N+1}$.

$$
\begin{aligned}
Q_{N+1} & =Q_{N}-z(b+N-a) K(a, b+N, z) Q_{N-1} \\
& \geqslant z Q_{N-1}+(b)_{N}-z(b+N) \frac{1}{b+N} Q_{N-1}+a z K(a, b+N, z) Q_{N-1} \\
& \geqslant(b)_{N}+a z^{N} K(a, b+N, z)
\end{aligned}
$$

which completes the proof of the theorem.

The truncation error (4.10) can now readily be estimated. For small values of $z$ we use (4.4), (4.12) and (4.13) to obtain

$$
\left|K(a, b, z)-P_{N}\right| Q_{N}|\leqslant|(b-a+1)_{N} \mid z^{N} /(b)_{N}(b)_{N+1} .
$$

For large values of $z$ we can write

$$
\left|K(a, b, z)-P_{N} / Q_{N}\right| \leqslant\left|(b-a+1)_{N}\right| / a\left\{(b)_{N}+z^{N}\right\} .
$$

These estimates show that the expansion (4.6) simultaneously corresponds to the Taylor expansion of the right-hand side of (4.2) and to its asymptotic expansion as $z \rightarrow \infty$. Obviously, this also applies to the expansion (2.6) since $F(p, x)$ is a special case of the function $K$ by virtue of Eq. (4.3). For this special case $(b=a)$ we can derive an exact formula for the truncation error (4.10) in terms of the confluent hypergeometric function $M$.

THEOREM 2. With $a$ and $z$ satisfying (4.2) and $b=a$ the denominators $Q_{n} d e-$ fined by (4.11) can be written as

$$
\begin{gathered}
Q_{n}=(a)_{n} M(-n, 1-a-n, z) \quad(n=0,1, \ldots, N), \\
Q_{N+1}=(a)_{N} / M(N, a+N,-z) .
\end{gathered}
$$

Proof. It is to be understood that the right-hand side of (4.17) always represents a polynomial in $z$ of degree $n$. If we take $\alpha=1-n, \beta=2-a-n$ in the relation (3.2) then these polynomials are found to satisfy 


$$
\begin{aligned}
(a+n-2) & (a+n-1) M(-n, 1-a-n, z) \\
= & (a+n-2)(a+n-1+z) M(1-n, 2-a-n, z) \\
& -z(n-1) M(2-n, 3-a-n, z) .
\end{aligned}
$$

We multiply this equation by $(a)_{n-2}$ and introduce

$$
Q_{n}^{*}=(a)_{n} M(-n, 1-a-n, z),
$$

whence

$$
Q_{n}^{*}=(a+n-1+z) Q_{n-1}^{*}-z(n-1) Q_{n-2}^{*} .
$$

It is seen that both $Q_{n}^{*}$ and $Q_{n}$ satisfy the second order recurrence relation (4.11) since $b=a$. Moreover, from (4.11) and (4.19) $Q_{0}^{*}=Q_{0}, Q_{1}^{*}=Q_{1}$ so that

$$
Q_{n}^{*}=Q_{n} \quad(n=0,1, \ldots, N)
$$

which proves (4.17). With the result (4.17) and the definitions (4.11) and (4.2) we obtain for $b=a$

$$
\begin{aligned}
Q_{N+1}= & (a)_{N} M(-N, 1-a-N, z) \\
& -z N \frac{M(a, a+N+1, z)}{(a+N) M(a, a+N, z)}(a)_{N-1} M(1-N, 2-a-N, z) .
\end{aligned}
$$

The quotient of the two functions $M$ can be rewritten with Kummer's transformation which yields

$$
Q_{N+1}=(a)_{N-1} V /(a+N) M(N, a+N,-z),
$$

where

$$
\begin{aligned}
V= & (a+\grave{N})(a+N-1) M(N, a+N,-z) M(-N, 1-a-N, z) \\
& -N z M(1-N, 2-a-N, z) M(N+1, a+N+1,-z) .
\end{aligned}
$$

Provided that $a \neq$ integer, the expression $V$ equals $(a+N)(a+N-1)$ by virtue of Eq. (3.4). Hence

$$
Q_{N+1}=(a)_{N} / M(N, a+N,-z), \quad a \neq 1,2,3, \ldots .
$$

A continuity argument shows that this result is also correct in case $a=1,2,3, \ldots$, which proves (4.18) and hence the theorem.

With the results of this theorem we can easily find an expression for the truncation error of the expansion (2.6) for $F(p, x)$. From Eqs. (4.3), (4.10), (4.2) and Theorem 2 this is found to be

$$
F(p, x)-a x \frac{P_{N}}{Q_{N}}=\frac{a(1)_{N}}{(a+N)(a)_{N}(a)_{N}} \frac{M(a, a+N+1, z)}{M(a, a+N, z)} \frac{M(N, a+N,-z)}{M(-N, 1-a-N, z)} x z^{N},
$$

where $z=x^{p}, a=1 / p$ as before.

This holds for every $a>0$, provided that $M(-N, 1-a-N, z)$ is always interpreted as a polynomial in $z$ of degree $N$. An application of Kummer's transformation 
yields

$$
\begin{aligned}
F(p, x)-a x \frac{P_{N}}{Q_{N}} & =\frac{N !}{(a)_{N}(a+1)_{N}} \frac{M(a, a+N+1, z)}{M(-N, 1-a-N, z)} x z^{N} e^{-z} \\
& \sim \frac{N !}{(a)_{N}(a+1)_{N}} x z^{N} e^{-2 z},
\end{aligned}
$$

where the asymptotic behavior for large values of $N$ follows from the limits

$$
\lim _{N \rightarrow \infty} M(a, a+N+1, z)=1, \quad \lim _{N \rightarrow \infty} M(-N, 1-a-N, z)=e^{z}
$$

Note that the result (4.20) does not imply that the truncation error is exponentially small as $z \rightarrow \infty$ since the double limit $N \rightarrow \infty, z \rightarrow \infty$ is nonuniform. In fact, the error decays algebraically as $z \rightarrow \infty$ analogous to (4.16). With the restriction $a \neq$ integer another application of Kummer's transformation to (4.20) produces

$$
\begin{array}{r}
F(p, x)-a x \frac{P_{N}}{Q_{N}}=\frac{N !}{(a)_{N}(a+1)_{N}} \frac{M(a, a+N+1, z)}{M(1-a, 1-a-N,-z)} x z^{N} e^{-2 z} \\
\quad \begin{array}{r}
a \neq 1,2,3, \ldots .
\end{array}
\end{array}
$$

For the special case $a=1 / 2$, the asymptotic behavior (4.20) and the result (4.21) coincide with the results of McCabe [2] for Dawson's integral. McCabe also investigated the quality of the asymptotic estimate (4.20) as compared with the true truncation error for the case $a=1 / 2$.

V. Applications. Apart from Dawson's integral (1.2), an interesting application of the expansion (2.6) is the case $p=3$, viz.

$$
F(3, x)=e^{-x^{3}} \int_{0}^{x} e^{t^{3}} d t
$$

which occurs in viscous fluid mechanics. The continued fraction (2.6) is an efficient method to evaluate this function for all $x \geqslant 0$ and the convergence of the expansion is fast for both small and large values of $x$. Note that the expansion (2.6) can be calculated without the use of exponential functions. Another application is the accurate computation of the remainder in the Taylor series

$$
e^{-z}=\sum_{i=0}^{n-1} \frac{(-z)^{i}}{i !}+\frac{(-1)^{n}}{n !} F\left(\frac{1}{n}, x\right), \quad x=z^{n}, n=1,2,3, \ldots
$$

A direct evaluation of the remainder as the difference of $\exp (-z)$ and the sum will be incorrect for $z$ small and $n$ large since this is subject to loss of significant digits. On the other hand, the continued fraction (2.6) for $F(1 / n, x)$ converges faster as $n$ gets large and/or $x$ becomes small.

To demonstrate the efficiency of the expansion (2.6) the following table shows the number of convergents of the expansion (2.6) which is needed to reach an accuracy of 10 significant digits. The table was calculated on an IBM 360/50 working in double precision. 


\begin{tabular}{rrrrrrrrrr}
\hline$z a$ & 10 & 5 & 3 & 2 & 1 & .5 & $1 / 3$ & .2 & .1 \\
\hline 0.1 & 5 & 6 & 6 & 7 & 8 & 8 & 8 & 9 & 9 \\
0.2 & 5 & 6 & 7 & 8 & 9 & 9 & 10 & 10 & 10 \\
0.5 & 6 & 8 & 9 & 10 & 11 & 12 & 12 & 13 & 13 \\
1.0 & 7 & 9 & 10 & 11 & 13 & 14 & 15 & 15 & 16 \\
2.0 & 8 & 10 & 12 & 14 & 16 & 18 & 19 & 19 & 20 \\
3.0 & 9 & 12 & 14 & 16 & 19 & 21 & 22 & 22 & 23 \\
5.0 & 10 & 13 & 16 & 19 & 22 & 25 & 26 & 27 & 28 \\
10 & 11 & 15 & 19 & 23 & 28 & 32 & 33 & 35 & 37 \\
20 & 11 & 14 & 16 & 19 & 26 & 34 & 38 & 41 & 44 \\
30 & 10 & 12 & 13 & 14 & 15 & 15 & 16 & 16 & 16 \\
40 & 10 & 11 & 11 & 12 & 12 & 12 & 12 & 12 & 12 \\
60 & 9 & 9 & 10 & 10 & 10 & 10 & 10 & 10 & 10 \\
100 & 8 & 8 & 8 & 8 & 8 & 8 & 8 & 8 & 8 \\
\hline
\end{tabular}

Acknowledgement. A part of this work was done while the author was visiting University College, London, on a combined grant from the British Science Research Council and the Netherlands Organization for the Advancement of Pure Research (Z.W.O.).

\footnotetext{
Department of Mathematics Technical University Twente P.O.B. 217

Enschede, The Netherlands
}

1. M. ABRAMOWITZ \& I. A. STEGUN (Editors), Handbook of Mathematical Functions, With Formulas, Graphs and Mathematical Tables, Nat. Bur. Standards, Appl. Math. Ser., no. 55, Superintendent of Documents, U. S. Government Printing Office, Washington, D. C., 1966. MR 34 \#8607.

2. J. H. MCCABE, "A continued fraction expansion, with a truncation error estimate, for Dawson's integral," Math. Comp., v. 28, 1974, pp. 811-816. MR 51 \#7243.

3. P. WYNN, "The numerical efficiency of certain continued fraction expansions. IA, IB," Nederl. Akad. Wetensch. Proc. Ser. A, v. 65 = Indag. Math., v. 24, 1962, pp. 127-137, 138-148. MR 25 \#2690a, b. 